\title{
Egresos por lesiones externas en un hospital de Ciudad Juárez, México
}

\author{
Beatriz A. Díaz-Apodaca, ${ }^{1}$ Federico G. De Cosio, ${ }^{2}$ \\ Gustavo Moye-Elizalde ${ }^{1}$ y Felipe F. Fornelli-Laffon ${ }^{1}$
}

Forma de citar Díaz-Apodaca BA, De Cosio FG, Moye-Elizalde G, Fornelli-Laffon FF. Egresos por lesiones externas en un hospital de Ciudad Juárez, México. Rev Panam Salud Publica. 2012;31(5):443-6.

RESUMEN En Ciudad Juárez (Chihuahua), México, la morbilidad y la mortalidad por lesiones aumentaron en forma alarmante a partir de 2008. El presente trabajo se propone determinar los cambios en el número de egresos hospitalarios por causas externas registrados durante el período 20082010 en un hospital de dicha ciudad. Se realizó un estudio retrospectivo descriptivo sobre la incidencia de causas externas como causa de egreso hospitalario en los años antes mencionados en el Hospital General Ciudad Juárez. La media de egresos hospitalarios por causas externas fue de $27 \%$, con el grupo etario de 25 a 44 años como el más afectado. Las fracturas fueron la causa de más de la mitad de los egresos. Los egresos hospitalarios por lesiones en Ciudad Juárez presentan una incidencia casi cuatro veces mayor que la reportada a nivel nacional.

Palabras clave Violencia; heridas y traumatismos; lesiones; heridas por arma de fuego; México.

Cada año las causas externas matan a más de 5 millones de personas, número que representa $9 \%$ de todas las muertes en el mundo. Noventa por ciento de ellas ocurren en países de ingresos bajos y medios. Una gran proporción de quienes sobreviven a los traumatismos sufren discapacidad temporal o permanente. Se estima que los traumatismos son responsables de 6 millones de años de vida con discapacidad $(1,2)$.

Se entiende por traumatismo a una lesión en el cuerpo, resultado de una exposición aguda a energía mecánica, térmica, eléctrica o química, o a radia-

\footnotetext{
Universidad Autónoma de Ciudad Juárez, Ciudad Juárez, Chihuahua, México. La correspondencia se debe dirigir a Beatriz A. Díaz-Apodaca. Correo electrónico: bdiaz@uacj.mx

2 Representación de la Organización Panamericana
} de la Salud en Belice. ción, en cantidades que excedan el límite de tolerancia fisiológico (3). Las causas externas de lesiones se clasifican en no intencionales e intencionales. Las lesiones por asalto o por violencia autoinfligida, y por guerra - o situaciones similares- son consideradas traumatismos intencionales (4). Las lesiones son las causas líderes de muerte y discapacidad en el mundo (5). De acuerdo a la Organización Mundial de la Salud (OMS), en el año 2000 las causas más comunes de días perdidos por discapacidad eran los accidentes de tránsito (22\%), las caídas (11\%), las lesiones autoinfligidas (11\%) y la violencia interpersonal (9\%) (6). Polinder y colaboradores reporta que los traumatismos de mayor incidencia en 10 países europeos fueron las lesiones superficiales, seguidas por fractura de rodilla/pierna y lesión de cráneo/ cerebro (7).
En el caso de México, en 2002 las causas externas representaron $7,3 \%$ del total de los egresos hospitalarios $-14,8 \%$ de hombres y $3,9 \%$ de mujeres. La causa más frecuente de lesiones en estos establecimientos fueron las fracturas $(44,9 \%)$, los traumatismos $(19,9 \%)$ y las heridas $(8,2 \%)(8)$.

Entre 2008 y 2010 Ciudad Juárez (Chihuahua) sufrió una ola de violencia similar a la de situaciones de guerra en las cuales los números de muertes y lesiones, especialmente por armas de fuego, experimentaron aumentos marcados. En 2007 el número de muertes violentas fue de 301; en 2008, 1 587; en 2009, 2 643, y en 2010, 3 037, con tasas de 22,6, 119,1, 198,4 y 228 por 100000 habitantes, respectivamente. Esta violencia también se ha visto reflejada en el número de egresos por lesiones de causas externas en los diferentes hospitales de la ciudad. 
El presente estudio tiene como objetivo determinar el número de egresos por causas externas durante el período 2008-2010 en un hospital público de una ciudad fronteriza mexicana expuesta a violencia extrema desde 2008.

\section{Metodología y análisis}

Se realizó un estudio retrospectivo descriptivo en el Hospital General de Ciudad Juárez (HGCJ) sobre la incidencia de causas externas en los egresos de este establecimiento durante los años 2008, 2009 y 2010. Se incluyeron 4844 casos, de los cuales $178(3,6 \%)$ fueron descartados por carecer de información clave para este trabajo, quedando solo 4666 casos. El HGCJ es un hospital público de primera elección en casos de traumatismos, sobre todo de personas sin seguridad social, derechohabientes del Seguro Popular y empleados del gobierno estatal.

Previo a la recolección de la información, el protocolo de investigación fue presentado a los Comités de Ética de la Universidad Autónoma de Ciudad Juárez y del HGCJ para su revisión y autorización. Con el fin de mantener la confidencialidad de las personas afectadas, solo se incluyó información proveniente de la base de datos de egresos del HGCJ tomada de los expedientes. Los datos facilitados por el HGCJ sirvieron para estimar la frecuencia de egresos por traumatismos intencionales y no intencionales durante el período 1 de enero de 2008 a 31 diciembre de 2010. Las variables obtenidas fueron sexo, edad, año de egreso y causa de egreso. Las lesiones como causa de egreso y con diagnóstico de fracturas, heridas, luxaciones, esguinces y torceduras, quemaduras, cuerpos extraños, envenenamientos y amputaciones fueron clasificadas utilizando la $10^{\text {a }}$ Clasificación Internacional de Enfermedades (1). El análisis de la base de datos se realizó con el paquete estadístico STATA, versión 12.

Con las variables cualitativas se utilizó el análisis univariado para obtener frecuencias absolutas y relativas, en tanto que la información se presentó en tablas de frecuencias. Para la edad, única variable continua, se utilizó la media como medida de tendencia central y la desviación estándar como medida de dispersión. Se utilizó t-student para evaluar las diferencias por sexo en los años de los estudios y análisis de la varianza (ANOVA, por sus siglas en inglés) para evaluar la diferencia en la prevalencia de las lesiones por año de ocurrencia y diferencia en las edades de los participantes en los años del estudio.

\section{Resultados}

De los 4666 casos analizados, la media de egresos hospitalarios por causas externas fue de $27 \%$ del total de los egresos en el período del estudio. Los 1693,1632 y 1519 egresos registrados por lesiones de causa externa en los años 2008, 2009 y 2010 representaron tasas de 119,1, 198,4 y 228 por 100000 habitantes, respectivamente, es decir incrementos de 5, 9 y 10 veces comparadas con la de 22,6 por 100000 habitantes en 2007 (cuadro 1). La razón hombres/mujeres con lesiones fue 4:1 $(P=<0,001)$. En los tres años estudiados se modificó la proporción de personas egresadas por lesiones de causas externas según grupo de edad, observándose un incremento de los egresos en los grupos de 25 a 44 y de 45 a 64 años de edad (cuadro 2).

Al analizar la incidencia de lesiones por causas externas de acuerdo al sexo, se halló que en 2008 la mayoría de las lesiones en las mujeres se presentaron en los extremos de la vida, mientras que en 2009 y 2010 el grupo etario más frecuente fue el de 25 a 44 años. Con relación a los hombres, la mayoría de los egresos pertenecieron al grupo en edad productiva (15 a 44 años), con un incremento constante. Los hombres fueron significativamente más jóvenes que las mujeres en cada uno de los años del estudio ( $P=<0,001$ ) (cuadro 3 ).

Las fracturas causaron poco más de la mitad de los egresos hospitalarios de lesiones por causas externas, seguidas por heridas y traumatismos. Al separar por sexo, las primeras tres causas de egresos fueron las mismas en hombres que en mujeres, pero un porcentaje mayor de mujeres que hombres presentaron fracturas $(67,5 \%$ y $47 \%)$, y los hombres sufrieron tres veces más heridas, y dos veces más amputaciones, que las mujeres (cuadro 4). Los hombres fueron los más afectados por fracturas (razón 3:1). Las fracturas más frecuentes en ambos sexos fueron pierna $(51,4 \%)$, antebrazo $(25,6 \%)$ y fémur $(17,0 \%)$. Al separar por sexo, las fracturas en los hombres no se modificaron, pero en las mujeres la de

CUADRO 1. Egresos hospitalarios y por causas externas en el Hospital General de Ciudad Juárez, Chihuahua, México, 2008-2010

\begin{tabular}{|c|c|c|c|c|c|c|c|}
\hline \multirow[b]{3}{*}{ Egresos } & \multicolumn{7}{|c|}{ Año } \\
\hline & \multicolumn{2}{|c|}{2008} & \multicolumn{2}{|c|}{2009} & \multicolumn{2}{|c|}{2010} & \multirow[b]{2}{*}{$P^{a}$} \\
\hline & No. & $\%$ & No. & $\%$ & No. & $\%$ & \\
\hline \multicolumn{8}{|l|}{ Con diagnóstico de } \\
\hline causas externas & 1693 & 27,9 & 1632 & 25,4 & 1519 & 27,8 & $<0,001$ \\
\hline Otros & 4372 & 72,1 & 4800 & 74,6 & 3953 & 72,2 & \\
\hline Total & 6065 & 100 & 6432 & 100 & 5472 & 100 & \\
\hline
\end{tabular}

Fuente: elaboración de los autores.

a Análisis de la varianza (ANOVA, por sus siglas en inglés).

CUADRO 2. Egresos hospitalarios debidos a causas externas, por grupos de edad, Hospital General de Ciudad Juárez, Chihuaha, México, 2008-2010

\begin{tabular}{|c|c|c|c|c|c|c|c|}
\hline \multirow{3}{*}{$\begin{array}{c}\text { Grupo de edad } \\
\text { (años) }\end{array}$} & \multicolumn{7}{|c|}{ Año } \\
\hline & \multicolumn{2}{|c|}{2008} & \multicolumn{2}{|c|}{2009} & \multicolumn{2}{|c|}{2010} & \multirow[b]{2}{*}{$P^{a}$} \\
\hline & No. & $\%$ & No. & $\%$ & No. & $\%$ & \\
\hline $0-14$ & 361 & 21,3 & 330 & 20,2 & 163 & 10,7 & \\
\hline 15-24 & 384 & 22,7 & 372 & 22,8 & 358 & 23,6 & \\
\hline 25-44 & 586 & 34,6 & 622 & 38,1 & 614 & 40,4 & $<0,001$ \\
\hline $45-64$ & 199 & 11,7 & 207 & 12,7 & 270 & 17,8 & \\
\hline$\geq 65$ & 163 & 9,6 & 101 & 6,2 & 114 & 7,5 & \\
\hline Total & 1693 & 100 & 1632 & 100 & 1519 & 100 & \\
\hline
\end{tabular}

Fuente: elaboración de los autores.

a Análisis de la varianza (ANOVA, por sus siglas en inglés). 
CUADRO 3. Incidencia de traumatismos intencionales y no intencionales, por grupo de edad y sexo, Hospital General de Ciudad Juárez, Chihuaha, México, 2008-2010

\begin{tabular}{|c|c|c|c|c|c|c|c|c|c|}
\hline \multirow[b]{3}{*}{$\begin{array}{l}\text { Grupo de edad } \\
\text { (años) }\end{array}$} & \multicolumn{9}{|c|}{ Año } \\
\hline & \multicolumn{3}{|c|}{2008} & \multicolumn{3}{|c|}{2009} & \multicolumn{3}{|c|}{2010} \\
\hline & $\begin{array}{c}\text { Total } \\
(n=1693) \\
\%\end{array}$ & $\begin{array}{c}\text { Hombres } \\
(n=1354) \\
\%\end{array}$ & $\begin{array}{c}\text { Mujeres } \\
(n=339) \\
\%\end{array}$ & $\begin{array}{c}\text { Total } \\
(n=1632) \\
\%\end{array}$ & $\begin{array}{c}\text { Hombres } \\
(n=1274) \\
\%\end{array}$ & $\begin{array}{c}\text { Mujeres } \\
\left(\begin{array}{c}n=358) \\
\%\end{array}\right.\end{array}$ & $\begin{array}{c}\text { Total } \\
(n=1519) \\
\%\end{array}$ & $\begin{array}{c}\text { Hombres } \\
(n=1149) \\
\%\end{array}$ & $\begin{array}{c}\text { Mujeres } \\
(n=370) \\
\%\end{array}$ \\
\hline $0-14$ & 21,3 & 19,1 & 30,1 & 20,2 & 18,6 & 25,1 & 10,7 & 9,5 & 15,1 \\
\hline $15-24$ & 22,7 & 25,9 & 10,0 & 22,8 & 26,1 & 11,5 & 23,6 & 26,0 & 16,2 \\
\hline $25-44$ & 34,6 & 37,9 & 20,4 & 38,1 & 40,3 & 30,2 & 40,4 & 43,9 & 29,7 \\
\hline $45-64$ & 11,7 & 11,7 & 11,5 & 12,7 & 11,3 & 18,2 & 17,8 & 16,7 & 21,4 \\
\hline$\geq 65$ & 9,6 & 5,3 & 26,8 & 6,2 & 3,7 & 14,8 & 7,5 & 4,0 & 18,4 \\
\hline
\end{tabular}

Fuente: elaboración de los autores.

CUADRO 4. Causas externas de egreso más frecuentes, por sexo, Hospital General de Ciudad Juárez, Chihuaha, México, 2008-2010

\begin{tabular}{|c|c|c|c|c|c|c|c|}
\hline \multirow[b]{2}{*}{ Causa } & \multicolumn{2}{|c|}{ Total } & \multicolumn{2}{|c|}{ Hombres } & \multicolumn{2}{|c|}{ Mujeres } & \multirow[b]{2}{*}{$P a$} \\
\hline & No. & $\%$ & No. & $\%$ & No. & $\%$ & \\
\hline Fractura & 2398 & 51,4 & 1725 & 47,0 & 672 & 67,5 & \\
\hline Heridas & 1022 & 21,9 & 940 & 25,6 & 85 & 8,5 & \\
\hline Traumatismos & 803 & 17,2 & 686 & 18,7 & 116 & 11,7 & \\
\hline \multicolumn{8}{|c|}{ Luxaciones, esguinces } \\
\hline $\mathrm{y}$ torceduras & 168 & 3,6 & 125 & 3,4 & 44 & 4,4 & $<0,001$ \\
\hline Quemaduras & 168 & 3,6 & 121 & 3,3 & 47 & 4,7 & \\
\hline Cuerpos extraños & 43 & 0,9 & 29 & 0,8 & 14 & 1,4 & \\
\hline Envenenamientos & 35 & 0,7 & 18 & 0,5 & 15 & 1,5 & \\
\hline Amputaciones & 29 & 0,6 & 26 & 0,7 & 3 & 0,3 & \\
\hline Total & 4666 & 100 & 3670 & 100 & 996 & 100 & \\
\hline
\end{tabular}

Fuente: elaboración de los autores.

a Chi cuadrado.

antebrazo fue la más frecuente, seguida por fractura de fémur y de pierna. Del total de las fracturas registradas (2 340), $404(17,3 \%)$ fueron fracturas expuestas secundarias a herida por proyectil de arma de fuego $-82,2 \%$ de ellas en hombres.

Once veces más hombres que mujeres sufrieron heridas en diferentes partes del cuerpo durante el período examinado, siendo las regiones abdominal/ lumbosacra/pélvica, tórax y cabeza las más afectadas. No se observó diferencia en frecuencia entre sexos en las tres primeras causas de egresos por heridas.

Los traumatismos fueron la tercera causa más frecuente de egresos, con el trauma intracraneal como responsable de $64 \%$ del total de los egresos, seguido por traumatismos múltiples y en regiones no especificadas. Más hombres que mujeres sufrieron traumatismo intracraneal.

\section{Conclusiones}

Una de las limitantes del presente trabajo es que no contó con el número de egresos por muertes debidas a causas externas, el cual hubiera permitido observar las variaciones que se pudieran haber dado en la proporción de morbilidad y mortalidad durante los tres años analizados. Asimismo, la información presentada corresponde a un solo hospital público y no se incluye ninguno de los nosocomios de seguridad social de la ciudad, los cuales se supone que deben presentar cambios similares en la frecuencia de sus egresos. Otra limitante es la escasa bibliografía que documente eventos de violencia sin estar en situación de guerra como la que se vive actualmente en Ciudad Juárez. No fue posible conseguir información de cambios en la frecuencia de lesiones tan importante como la que se vive en Ciudad Juárez, ya que este tipo de situaciones solo se presentan en guerras.

Los resultados del estudio constatan un considerable incremento en los egresos por causas externas en un hospital público en una ciudad mexicana expuesta a violencia extrema: las tasas de egreso en 2008, 2009 y 2010 fueron 5, 9 y 10 veces más altas que la de 22,6 por
100000 habitantes registrada en 2007. También revelaron que los egresos hospitalarios por lesiones debidas a causas externas durante el período examinado registraron una pequeña disminución en los hombres: $80 \%$ en $2008,78,1 \%$ en 2009 y $75,6 \%$ en 2010 . Tal reducción podría ser efecto secundario al incremento en el número de muertes violentas en el mismo período (de 1587 en 2008 a 3039 muertes en 2010), y significar que más lesionados mueren durante el traslado al hospital o que la causa del egreso fue defunción. No obstante, también se ha documentado un incremento en el número de mujeres que son víctimas directas de las violencia en la ciudad a medida que ocupan posiciones dejadas por hombres.

Otro factor que pudiera estar impactando en el significativo incremento de las tasas de egresos hospitalarios es el incremento en el número de personas que perdieron su trabajo durante el período de estudio por el cierre de un importante número de empresas de manufactura que emigraron a otras partes de México, menos violentas, o a otro país con mano de obra más barata y que favoreció el incremento en personas que perdieron su derecho-habiencia a servicios de salud y fueron atendidos en una institución para población abierta.

La proporción de causas externas como egresos hospitalarios tuvo una razón hombre/mujer de 4:1, similar a la de egresos nacionales pero mucho mayor que la reportada por la OMS (2:1). También se halló que el grupo etario más afectado por lesiones es el de 15 a 44 años (5).

La mayoría de los egresos hospitalarios por lesiones debidas a causas externas fueron las fracturas, en coincidencia con lo informado en otro estudio llevado a cabo en México, aunque en el presente 
trabajo la proporción fue mayor: $51,4 \%$ vs. $44,9 \%$ (7). La segunda causa de egreso fueron las heridas de todo tipo $(21,9 \%)$ y la tercera los traumatismos $(17,2 \%)$, orden que se invierte a nivel nacional con $8,1 \%$ y $19,9 \%$ respectivamente. Más hombres que mujeres sufrieron lesiones cráneoencefálicas, lo que concuerda con el trabajo de García-Gómez y colaboradores (9) realizado en un hospital de La Habana.
Este es el primer trabajo sobre causas externas relacionadas con egresos hospitalarios realizado en un período con un marcado incremento en la violencia, en particular las lesiones por armas de fuego que afectan sobre todo a los hombres. Sus resultados muestran la frecuencia de las lesiones en una ciudad que ha estado expuesta a violencia extrema sin que haya situación de guerra y para

\section{REFERENCIAS}

1. World Health Organization. International Classification of External Causes of Injury (ICECI). Disponible en: http://www.who. int/classifications/icd/adaptations/iceci/ en/.htm Acceso el 26 de febrero de 2012.

2. World Health Organization. Health Topics 2011: Injuries. Disponible en: http:who.int/ topics/injuries/about/en/index.html Acceso el 26 de febrero de 2012.

3. Organización Panamericana de la Salud. Prevención de la violencia y los traumatismos y promoción de la seguridad: un llamado a la acción en la Región. Washingon, D.C.: OPS; 2008. Disponible en http://www.paho.org/ spanish/gov/cd/CD48.r11-s.pdf Acceso el 26 de febrero de 2012.
4. Baker SP, O'Neil B, Karpf RS. The injury fact book. Lexington, U.S.A.: Lexington Books; 1984.

5. Krug EG, Sharma GK, Lozano R. The global burden of injuries. Am J Public Health. 2000; 90(4):523-6.

6. World Health Organization. The injury chartbook. A graphical overview of the global burden of injuries. Ginebra, Suiza: WHO; 2002.

7. Polinder S, Meerding WJ, van Baar ME, Toet $\mathrm{H}$, Mulder S, van Beeck EF, et al. Cost estimation of injury-related hospital admissions in 10 European countries. J Trauma. 2005;59(6): 1283-91.

8. Secretaría de Salud de México. Estadística de egresos hospitalarios del sector público del la cual las instituciones hospitalarias no estaban preparadas. Podría ser muy positivo que esta experiencia sirviera para elaborar protocolos de diagnóstico, tratamiento y rehabilitación destinados a quienes sufren estas lesiones, así como para hacer seguimiento de las tendencias que registran dichos actos de violencia.
Sistema Nacional de Salud. 2003. Dirección General de Información y Evaluación del Desempeño, de la Secretaría de Salud Pública de México. 2004;46(5):484-7.

9. García Gómez A, González Corrales Ll, Gutiérrez Gutiérrez L, Trujillo Machado V, López González JC. Caracterización del traumatismo cráneo-encefálico grave. Rev $\mathrm{Cub}$ Med Mil. 2009;38(3-4):10-7.

Manuscrito recibido el 22 de agosto de 2011. Aceptado para publicación, tras revisión, el 23 de febrero de 2012.

ABSTRACT In Ciudad Juarez, Chihuahua, Mexico, morbidity and mortality from injuries have increased alarmingly since 2008 . This paper aims to examine the changes in the number of hospital discharges for external injuries recorded during the 2008-2010 period in a hospital in Ciudad Juarez. A descriptive retrospective study conducted at the Ciudad Juarez General Hospital looked at the incidence of external injuries as the reason for hospital discharges during the period under analysis. The average proportion of hospital discharges attributed to external injuries was 27\%, with the 25-44-year-old age group being the most affected. More than half of the discharges were for fractures. The incidence rate of hospital discharges attributed to injuries in Ciudad Juarez was almost four times greater than that reported at the national level.

Key words Violence; wounds and injuries; injuries; wounds, gunshot; Mexico. 